\title{
Excitation and ionization of sodium in meteoroid impacts on the Moon
}

\author{
A. Carbognani ${ }^{1}$ and G. Cremonese ${ }^{2}$ \\ ${ }^{1}$ Department of Physics, University of Parma, Parco Area delle Scienze 7A, 43100 Parma, Italy \\ e-mail: albino@fis.unipr.it \\ 2 INAF - Osservatorio Astronomico Padova, Vic. Osservatorio 5, 35122 Padova, Italy \\ e-mail: cremonese@pd.astro.it
}

Received 19 April 2002 / Accepted 6 August 2002

\begin{abstract}
After a brief historical review about the Moon sodium exosphere and lunar impacts, the attention is focused on the lack of enhancements of the sodium emissions by meteor showers different from Leonids. In order to contribute to the solution of this problem we perform an order-of-magnitude calculation of the physical conditions of sodium atoms during meteoroid impacts. This calculation suggests that the lack of sodium emission enhancements during different meteor showers could be caused by the different ionization degree of the sodium atoms which, in turn, depends on the meteoroid impact velocity.
\end{abstract}

Key words. Meteors - Meteoroids - Moon - Telescope

\section{Introduction}

The Moon has a tenuous atmosphere containing atomic sodium and potassium discovered in 1988 (Potter \& Morgan 1988). These elements must be continuously resupplied since the Moon gravity is too weak to retain the atoms for more than a few hours. Several mechanisms have been suggested to explain the resupply: solar wind, micrometeoroid impacts, thermal desorption and photon-stimulated desorption (Smyth et al. 1995). However there is little or no consensus about the dominating mechanism.

In order to understand whether the micrometeoroids can influence the Moon exosphere, some observations have been taken during and after the 1997 passage of the Leonids (Hunten et al. 1998) to examine the lunar circumstances at the time of collision Moon-shower and looking for possible effects. The results showed a correlation between the lunar exosphere and the meteor shower, representing an interesting confirmation of two previous possible detections of meteor stream effects on the lunar sodium exosphere (Hunten et al. 1991; Verani et al. 1998). In the first case it was not possible to associate firmly the lunar observations to a known meteor shower while, in the second case, the enhancement of lunar exosphere was associated to the Leonids (in the 1995).

Further observations have been obtained during the 1998 Leonid passage from Smith et al. (1999). They utilized allsky images in the sodium light $(589.6,589.0 \mathrm{~nm})$, of the antisolar point, during the New Moon. Their observation took

Send offprint requests to: A. Carbognani, e-mail: albino@fis.unipr.it place three days around the Leonid peak and put into evidence a sodium spot changing its intensity. They defined such a feature as the bright sodium Moon-tail produced by Leonid impacts on the Moon surface.

Apart to the possible correlation between the Leonids and the lunar sodium exosphere observed on three different passages, no correlations with other showers have been observed. Specifically, no enhancements of the lunar exosphere have been detected during the 1999 Quadrantid meteor shower passage (Verani et al. 2001).

In the last few years other types of observations of the lunar surface have been performed during meteor showers, in order to improve the knowledge about the correlations already studied in the sodium light. Some videos registered during the 1999 and 2001 Leonid passages showed short light flashes on the lunar surface, with apparent visual magnitude between +3 and +4 (Ortiz et al. 2000; Bellot Rubio et al. 2000; Cudnik et al. 2002; Ortiz et al. 2002). These flashes have been interpreted as the result of meteoroid impacts on the Moon.

In this work we deal with the correlation between meteor showers and the lunar exosphere with respect to the sodium excitation and ionization degree (immediately after the meteoroid impact). We will analyze the sodium atom as it is one of the most abundant elements in the lunar exosphere, it has the highest resonant scattering interaction with the solar radiation and it is the only specie observed during the meteor showers. Only Smith et al. (1999) made all-sky images in the potassium light $(769.9 \mathrm{~nm})$, observing no emission.

This work will mainly help us to establish which meteor shower is more suitable to be observed in sodium light in order 
to define the contribution of meteoroid impacts to the Moon sodium exosphere. Then this study could be taken into account on planning sodium exosphere observations. At the moment we cannot say if this work may be interesting to study also the bright flashes as there is not a clear relation between them and the sodium atoms.

\section{Impact conditions}

The observation of lunar impacts in visible light is possible only if the meteoroids hit the night side of the Moon as seen from Earth, and this depends on the encounter geometry of the meteor shower with the Earth-Moon system.

The sodium emissions may be used as good tracers of the mechanisms working in the exosphere and during the impacts.

In the initial stage of an impact on the lunar surface the particles penetrate deeply into the soil, leading to its vaporization. The soil vapor temporary covers the hot meteoroid substance. Subsequently the expanding envelope of the target becomes transparent leading to the increase of the radiation flux caused by the meteoroid plasma. The peak of radiation flux occurs approximately $0.5-1 \mathrm{~ms}$ after the impact (Artem'eva et al. 2000).

Let $m$ be the meteoroid mass and $v$ the geocentric velocity. The kinetic energy of the body is:

$\epsilon=\frac{1}{2} m v^{2}$

In Eq. (1) the Moon orbital velocity is considered negligible with respect to the Earth orbital velocity. If $\tau$ is the fraction of kinetic energy partitioned in the plasma production, the temperature of plasma cloud is given by energy conservation:

$\epsilon \tau=\frac{3}{2} N k T$

where $k$ is the Boltzmann constant, and $N$ is the particle density of the impact-generated plasma:

$N=\frac{m}{<m>}$.

In Eq. (3) $<m>$ is the mean mass of meteoroid atoms, which depends on the chemical composition of the body. From Eqs. (1) and (3) we obtain:

$T=\frac{2 \epsilon \tau}{3 k N}=\frac{\tau<m>v^{2}}{3 k}$

it follows that, in first approximation, $T$ is independent from the mass $m$ of the meteoroid. The $N$ value from Eq. (3) is not the real particle density value, because also part of the lunar soil evaporates in the impact. Being the soil contribution negligible in hypervelocity impact, we can use $N$ without corrections.

Numerical simulation of Leonid impacts on the Moon showed that the typical temperature of the plasma cloud is $1 \mathrm{eV}$ or $11600 \mathrm{~K}$ for a meteoroid density of $1000 \mathrm{~kg} / \mathrm{m}^{3}$ (Artem'eva et al. 2001). Comparing this value with Eq. (4) and considering $v=71 \mathrm{~km} \mathrm{~s}^{-1}$, we obtain $\tau \approx 0.0025$.

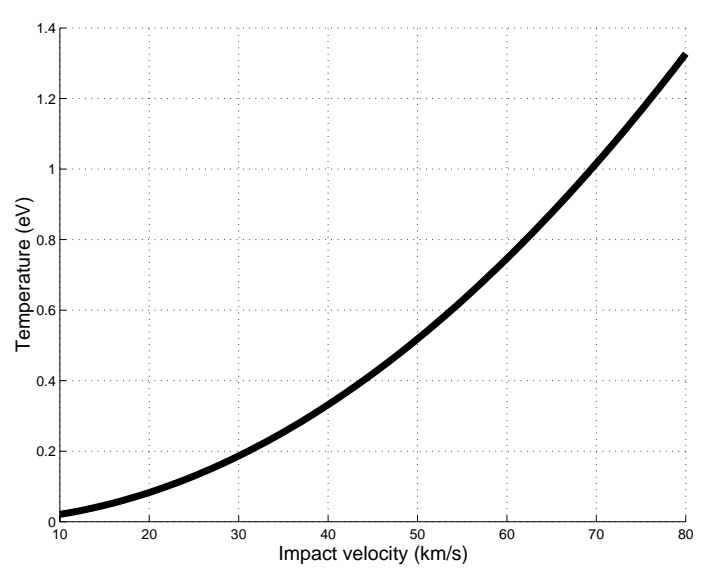

Fig. 1. Temperature of the plasma cloud immediately after the impact vs. impact velocity. The $\tau$ value is fixed in order to have $T=1 \mathrm{eV}$ for $v=71 \mathrm{~km} \mathrm{~s}^{-1}$. The interval of $v$ cover all the possible values for the Earth-Moon system.

Let $f$ be the fraction of sodium in the meteoroid mass and $m_{\mathrm{Na}}$ the mass of a single $\mathrm{Na}$ atom. Then, the total number of $\mathrm{Na}$ atoms is:

$N_{\mathrm{Na}}=\frac{m f}{m_{\mathrm{Na}}}$

For a typical chondrite composition $f \approx 0.01$ (Jarosewich 1990) and the mean atomic mass is $\langle m\rangle=24.5$ a.m.u. We consider $f$ between 0.001 and 0.1 , in order to cover a wider range of physical values. Fixed the $\tau$ and $<m>$ values, we can extrapolate $T$ soon after the impact as a function of the impact speed using Eq. (4) (see Fig. 1). Of course the $T$ value varies quickly with time.

\section{The population of excited and ionized $\mathrm{Na}$ atoms}

Using the Boltzmann and Saha equations, with the values of $T$ and $N_{\mathrm{Na}}$ given by Eqs. (4) and (5), we can calculate the distribution of excited and ionized $\mathrm{Na}$ atoms.

The Boltzmann and Saha equations can be applied on systems in local thermodynamic equilibrium as it happens, in first approximation, for the plasma cloud. In fact, the characteristic time of ionization and recombination processes does not exceed few millisecond: less than the characteristic time of the flash, which is about $0.02 \mathrm{~s}$ (Bellot Rubio et al. 2000). Fast varying conditions of temperature and density can produce departures from local thermodynamic equilibrium, but this problem is beyond the scope of this work.

The Boltzmann equation gives the ratio between the atom density numbers in the $i$ excitation state and the atoms in the fundamental state:

$\frac{n_{i}}{n_{1}}=p_{i}=\frac{g_{i}}{g_{1}} \exp \left(-E_{i} / k T\right)$

In Eq. (6), $g_{i}(i=1,2,3, \ldots)$ is the degeneracy of the $E_{i}$ energetic level ( $i=1$ is the fundamental state). For energetic levels and degeneracy of $\mathrm{Na}$ atoms see Table 1. 
Table 1. Excited states of $\mathrm{Na}$ (neutral atom).

\begin{tabular}{lccc}
\hline \hline Energy $(\mathrm{eV})$ & Level & Degeneracy $\left(g_{i}\right)$ & Emission $(\mathrm{nm})$ \\
\hline 2.1046 & 2 & 2 & 589.6 \\
2.1067 & 3 & 4 & 588.9 \\
3.2 & 4 & 2 & 387.7 \\
3.6 & 5 & 6 & 344.7 \\
\hline
\end{tabular}

In the case of ionized states, it is necessary to use the Saha equation that gives the ratio between the $i+1$ and the $i$ ionized atoms ( $i=0$ is the neutral atom):

$\frac{n^{i+1}}{n^{i}}=\frac{2 z^{i+1}}{z^{i}} \frac{\left(2 \pi m_{\mathrm{e}} k T\right)^{3 / 2}}{n_{\mathrm{e}} h^{3}} \exp \left(-E^{i} / k T\right)$

where $n_{\mathrm{e}}$ is the electron volume density, $m_{\mathrm{e}}=9.1 \times 10^{-31} \mathrm{~kg}$ is the electron mass, $h=6.626 \times 10^{-34} \mathrm{~J} \cdot \mathrm{s}$ is the Planck constant, $E^{i}$ is the ionization energy and $z^{i}$ is the partition function of the $i$-ionized atom:

$z^{i}=g_{0}^{i}+\sum_{j=1}^{+\infty} g_{j}^{i} \exp \left(E_{j}^{i} / k T\right)$.

In Eq. (8) $g_{j}^{i}$ is the degeneracy of the excited $j$-level of an $i$-ionized atom, and $E_{j}^{i}$ is the corresponding energy. Both excitation and ionization energy are superior to $k T \approx 1 \mathrm{eV}$, so, in first approximation, the partition function is coincident with the degeneracy $g_{0}^{i}$ of the fundamental state of the atom $i$-ionized. The Saha equation becomes:

$\frac{n^{i+1}}{n^{i}} \simeq \frac{2 g_{i+1}}{g_{i}} \frac{\left(2 \pi m_{\mathrm{e}} k T\right)^{3 / 2}}{n_{\mathrm{e}} h^{3}} \exp \left(-E^{i} / k T\right)$.

In order to use Eq. (9), it is necessary to know the $n_{e}$ value. We may assume that the $\mathrm{Na}$ atoms are single ionized, because the second ionization energy is very high. In the numeric simulations of Leonid lunar impacts the maximum temperature of the plasma cloud is about $1 \mathrm{eV}$, comparable with the first ionization energy $\left(E^{0}=5.138 \mathrm{eV}\right)$, but much less than the second ionization energy $\left(E^{1}=47.290 \mathrm{eV}\right)$.

Adopting this approximation, the Saha equation is written only for $i=0$ and $n_{\mathrm{e}}=n^{1}$ : the free electrons number is equal to the $\mathrm{Na}$ atoms single ionized. Other alkaline metals (like potassium), with low ionization energy, are less abundant than sodium and other elements have higher ionization energy, so we can consider the sodium as the major contributor to the free electron population.

Now, we can calculate the ionization degree of sodium. If $n$ is the density particles number, $N$ the total number of particles and $V$ the volume of plasma cloud, we have:

$\frac{n_{\mathrm{e}} n_{\mathrm{i}}}{n_{\mathrm{n}}}=\frac{N_{\mathrm{e}}}{V} \frac{\alpha_{\mathrm{i}}}{1-\alpha_{\mathrm{i}}}$

where the subscript e refers to the electrons, $\mathrm{i}$ to the ions, $\mathrm{n}$ to the neutral particles and $\alpha_{\mathrm{i}}=N_{\mathrm{i}} / N_{\mathrm{Na}}$ is the sodium ionization degree. As stated above, the electron number is:

$N_{\mathrm{e}}=\alpha_{\mathrm{i}} N_{\mathrm{Na}}$.

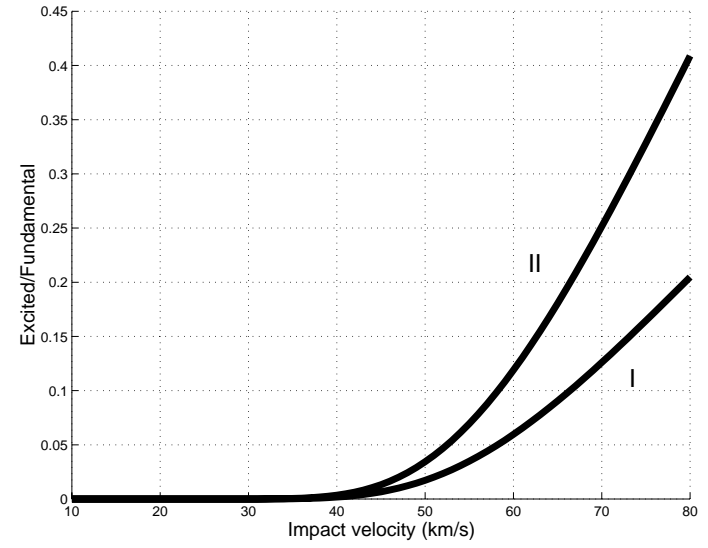

Fig. 2. Ratio between atoms in excited state and atoms in the fundamental state vs. impact velocity. The curve I is referred to the $2.1046 \mathrm{eV}$ level, while the curve II to the $2.1067 \mathrm{eV}$ level.

Using Eqs. (5), (10) and (11), Eq. (9) becomes:

$\frac{\alpha_{\mathrm{i}}^{2}}{1-\alpha_{\mathrm{i}}} \simeq \frac{m_{\mathrm{Na}}}{f \rho_{\mathrm{p}}} \frac{2 g_{1}}{g_{0}} \frac{\left(2 \pi m_{\mathrm{e}} k T\right)^{3 / 2}}{h^{3}} \exp \left(-E^{0} / k T\right)$

where $\rho_{\mathrm{p}}$ is the mean density of the plasma cloud, about $10^{-3}$ time the meteoroid density of $1000 \mathrm{~kg} / \mathrm{m}^{3}$ (Artem'eva et al. 2001). In this model, the dependence from the chemical composition is in the $f$ factor. Now we can calculate the ionization degree $\alpha_{\mathrm{i}}$ solving a simple second degree algebraical equation.

\section{Results}

The result for the sodium excited atoms is shown in Fig. 2. We have considered only the first two excited levels, responsible for the $D_{1}$ and $D_{2}$ emission lines (sodium doublet). The second level has a double population with respect to the first, because the degeneration is doubled (see Table 1). The population of the excited second level is never greater than $50 \%$ of the population in fundamental state. Only for impact velocity higher than $40 \mathrm{~km} \mathrm{~s}^{-1}$, the sodium excitation is not negligible.

The result for the ionization degree immediately after the impact is shown in Fig. 3. For meteoroids with low sodium concentration (but with the same impact velocity), the ionization degree of sodium is greater, because there are few free electrons available to recombine with ions. For $f<0.1$, the ionization degree is over $50 \%$ for impact velocity between 40 and $50 \mathrm{~km} \mathrm{~s}^{-1}$. For impact velocity lower than $40 \mathrm{~km} \mathrm{~s}^{-1}$ the sodium atoms are not much ionized, the same "critical velocity" already found in the two first excitation degrees. This result is weakly dependent by the plasma cloud density.

The impact intensity in the sodium doublet is proportional to $\left(p_{2}+p_{3}\right)\left(1-\alpha_{\mathrm{i}}\right)$. This function is shown in Fig. 4 for some $f$ values. Assuming a optically thin plasma cloud, the impact radiation flux $F$, received from Earth is:

$F=\frac{K N_{\mathrm{Na}}\left(1-\alpha_{\mathrm{i}}\right)\left(p_{2}+p_{3}\right) h \bar{v}}{4 \pi r^{2}}$.

In Eq. (13) $N_{\mathrm{Na}}$ is the number of sodium atoms, $K \approx 0.67$ the fraction of neutral atoms in the fundamental state, $h \bar{v}$ the mean 


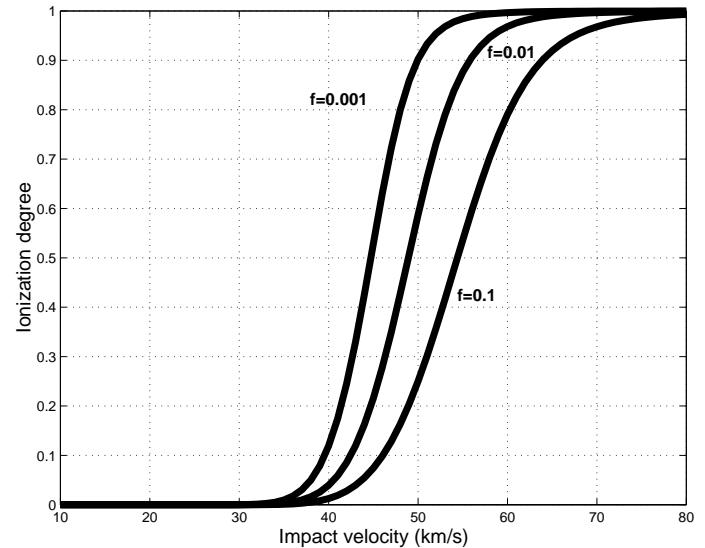

Fig. 3. Ionization degree of sodium atoms vs. impact velocity. Each curve is drawn for a different $f$ value.

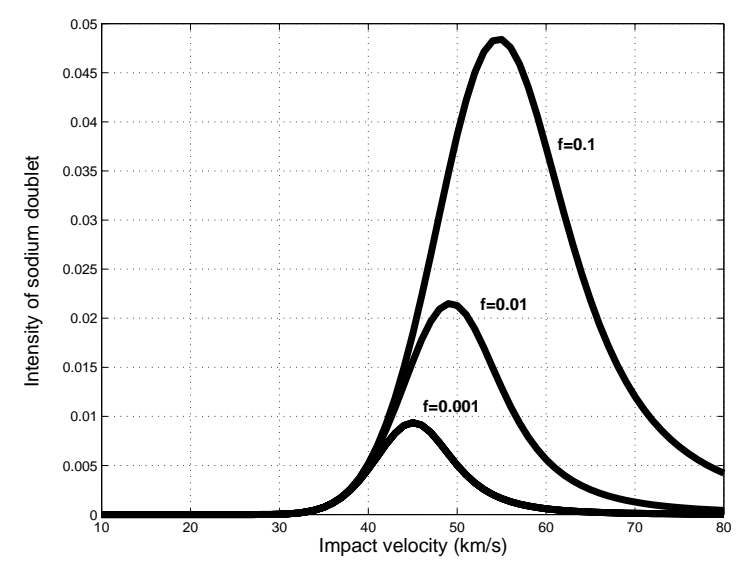

Fig. 4. Intensity (in arbitrary units) of sodium doublet vs. impact velocity for several $f$ values.

energy of a photon in the sodium doublet and $r$ the mean distance Earth-Moon. We may provide a rough estimate for the impact magnitude in sodium light using the Pogson's equation:

$m_{\text {impact }}=m_{\text {sun }}-2.5 \log _{10}\left(F / S_{\text {sun }}\right)$

where $m_{\text {sun }}=-26.74$ and $S_{\text {sun }}=1.85 \mathrm{~J} / \mathrm{s} \mathrm{m}^{2}$, are the apparent magnitude of the Sun and the solar constant in visible light. The result is shown in Fig. 5 for a meteoroid of a $100 \mathrm{~kg}$ mass. According to this result the impact detection in sodium light can be possible in the night side of the Moon using a mean diameter telescope in the best case.

There is an impact velocity where the intensity of sodium light is maximum (or magnitude is minimum). The velocities related to the maximum emission are in the range between 45 and $55 \mathrm{~km} \mathrm{~s}^{-1}$, increasing with the $f$ value. Assuming $f=0.1$, the major naked-eye meteor showers, with potentially observable sodium emission enhancements, are characterized by a geocentric velocity between $45-65 \mathrm{~km} \mathrm{~s}^{-1}$ (Lyrids, Perseids). Of course meteoroids with velocities as low as $40 \mathrm{~km} \mathrm{~s}^{-1}$ or as hight as $70 \mathrm{~km} \mathrm{~s}^{-1}$ will also produce emission in the $D_{1}$ e $D_{2}$ lines, although with lower efficiency. Lower $f$ values give lower sodium intensity in different geocentric velocity ranges as $43-55 \mathrm{~km} \mathrm{~s}^{-1}$ (Lyrids) for $f=0.01$ and $40-50 \mathrm{~km} \mathrm{~s}^{-1}$ (Quadrantids, Lyrids) for $f=0.001$.

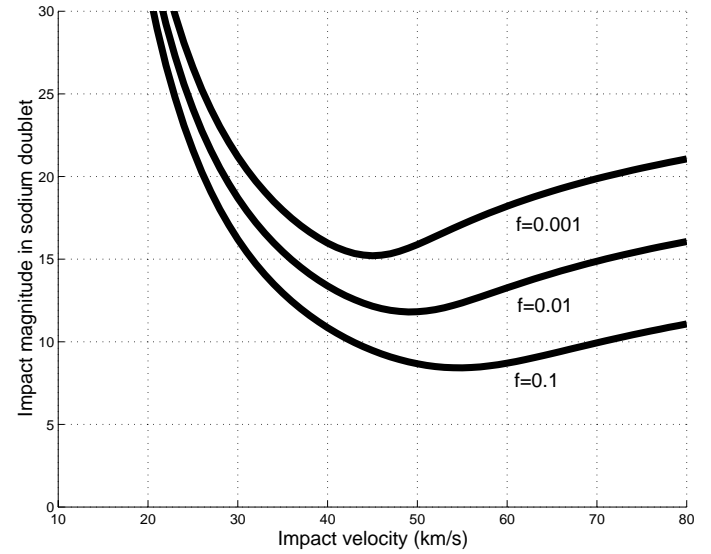

Fig. 5. Estimate of the impact magnitude in the sodium light vs. impact velocity for some $f$ values. The meteoroid mass is $100 \mathrm{~kg}$.

\section{Discussion}

The results of this work suggest the following mechanism to explain the lack of sodium emission enhancements by meteor showers different from Leonids. During the impact the meteoroid penetrates deeply into the lunar soil, leading to its vaporization. In the expansion of the cloud after the light flash the lunar dust grains mix with the meteoroid substance and adsorb the neutral sodium atoms and the ions survived at the recombination processes. On the dust grain surface the ionized atom can capture an electron to make a neutral sodium atom. The ionic radius is smaller than neutral radius, and the neutral adsorbed atom is at a smaller atom-surface separation than the equilibrium value. The configuration is repulsive and it can stimulate the desorption of the neutral $\mathrm{Na}$ atom. So, the recombined ions are free on the lunar exosphere and the real neutral atoms are bounded to the lunar dust. This repulsive process is similar to the photon-stimulated desorption of sodium from surface simulated in laboratory by Yakshinskiy \& Madey (1999) using lunar silicates.

The greater the impact velocity the greater the vaporized mass of lunar soil, and this helps the recombination ions processes. Taking into account this mechanism only the meteor showers characterized by high impact velocity can be the source of the exosphere sodium emission enhancements, assuming the same mass-fraction of sodium in the meteoroid. Meteor showers as Quadrantids and Geminids, with a meteoroid impact velocity of 41 and $36 \mathrm{~km} \mathrm{~s}^{-1}$, respectively, are too slow to produce high number of ionized sodium atoms (see Fig. 3).

After the Leonids, the meteor showers with highest impact velocity are $\eta$ Aquarids ( $\left.66 \mathrm{~km} \mathrm{~s}^{-1}\right), \theta-\alpha$ Aurigids $\left(66 \mathrm{~km} \mathrm{~s}^{-1}\right.$ ), Orionids $\left(66 \mathrm{~km} \mathrm{~s}^{-1}\right)$ and $\alpha$ Monocerontids $\left(65 \mathrm{~km} \mathrm{~s}^{-1}\right)$. However, there are no impact observations to date. The mass flux of a shower is an additional factor that can influence the sodium content of the lunar exosphere. If the mass flux is large also a shower with a low geocentric velocity could provide an amount of sodium atoms greater than a shower with higher velocity but with lower mass flux, assuming the same fraction $f$ of sodium. 
Further observations of the lunar sodium exosphere during different meteor showers can help us to refine the model, verifying the correlation between $f$ and the geocentric velocity suggested by the proposed mechanism, and to provide information on the composition of the meteoroids.

Acknowledgements. We thank Luigi Foschini for helpful discussions and the referee, L. R. Bellot Rubio, for his suggestions.

\section{References}

Artem'eva, N. A., Kosarev, I. B., Nemtchinov, I. V., Trubetskaya, I. A., \& Shuvalov, V. V. 2000, Sol. Sys. Res., 34, 453

Artem'eva, N. A., Kosarev, I. B., Nemtchinov, I. V., Trubetskaya, I. A., \& Shuvalov, V. V. 2001, Sol. Sys. Res., 35, 177

Bellot Rubio, L. R., Ortiz, J. L., \& Sada, P. V. 2000, Earth, Moon and Planets, 82-83, 575

Cudnik, B. M., et al. 2002, Lunar and Planet. Sci. Conf. XXXIII, Abstract 1329
Hunten, D. M., Kozlowski, R. W. H., \& Sprague, A. 1991, Geophys. Res. Lett., 18, 2101

Hunten, D. M., Cremonese, G., Sprague, A., et al. 1998, Icarus, 136, 298

Jarosewich, E. 1990, Meteoritics, 25, 323

Ortiz, J. L., Sada, P. V., Bellot Rubio, L. R., et al. 2000, Nature, 405, 921

Ortiz, J. L., Quesada, J. A., Aceituno, J., Aceituno, F. J., \& Bellot Rubio, L. R. 2002, ApJ, in press

Potter, A. E., \& Morgan, T. H. 1988, Science, 241, 675

Smith, S. M., Wilson, J. K., Baumgardner, J., \& Mendillo, M. 1999, Geophys. Res. Lett., 26, 1649

Smyth, W. H., \& Marconi, M. L. 1995, ApJ, 443, 371

Verani, S, Barbieri, C., Benn, C., \& Cremonese, G. 1998, Planet. Space Sci., 46, 1003

Verani, S, Barbieri, C., Benn, C., Cremonese, G., \& Mendillo, M. 2001, MNRAS, 327, 244

Yakshinskiy, B. V., \& Madey, T. E. 1999, Nature, 400, 642 